\section{Symptomatic cluster-like headache in Normal-Pressure Hydrocephalus (NPH) and possible arteritis: Case report and an overview of the literature on secondary cluster-like headaches}

\author{
Stefania Peruzzo and Carlo Lovati* \\ Headache Center, Neurology Unit, L. Sacco Hospital, University of Milan, Milan, Italy
}

\section{Background}

Cluster headache $(\mathrm{CH})$ is a primary headache belonging to the group of trigeminal-autonomic cephalalgias, characterized by short attacks of very severe headache, always involving the same side of the head, more frequently localized to the periorbital and temporal area, associated with homolateral tearing, red eye, nose obstruction or rhinorrhea, ptosis, miosis, and restlessness. These attacks generally last 15-180 minutes and can occur from once every day to 8 times a day.

Cluster headache affects about the $0.1 \%$ of the population and it is more frequent among males with an $\mathrm{M} / \mathrm{F}$ ratio of about $3 / 1$. The onset is generally observed in the young adulthood and its prevalence after the seventh decade is very low. In all the typical decades of onset, the $\mathrm{M} / \mathrm{F}$ ratio is about $3 / 1$, but onset after age 50 is more common in women, and over $20 \%$ of these late onset had a chronic form [1]. Moreover, women tend to develop $\mathrm{CH}$ at an earlier age, and are also more likely to show a second peak of $\mathrm{CH}$ incidence after the age of 50 [2].

Regardless, due to the presence of a typical decades of onset, is consistent to hypothesize that in non-typical age range, symptomatic forms may be more frequent.

A series of possible conditions have been described as causes of symptomatic cluster headaches as clearly reviewed in 2014 by Edvardsson B [3]. He summarized a series of conditions that may cause a cluster-like headache, term that seems more appropriate when clinical examination and/or radiological investigations put in evidence some abnormal element which may cause the headache.

These conditions include above all vascular disorders such as arterial aneurysms, arteriovenous malformations, cavernous hemangioma, subclavian steal syndrome, carotid artery

\section{More Information}

*Address for Correspondence: Carlo Lovati, Headache Center, Neurology Unit, L. Sacco Hospital, University of Milan, Milan, Italy, Email: carlo.lovati@asst-fbf-sacco.it

Submitted: 13 February 2020

Approved: 26 February 2020

Published: 27 February 2020

How to cite this article: Peruzzo S, Lovati C. Symptomatic cluster-like headache in NormalPressure Hydrocephalus (NPH) and possible arteritis: Case report and an overview of the literature on secondary cluster-like headaches. Insights Depress Anxiety. 2020; 4: 005-007.

DOI: dx.doi.org/10.29328/journal.ida.1001012

Copyright: @ 2020 Peruzzo S, et al. This is an open access article distributed under the Creative Commons Attribution License, which permits unrestricted use, distribution, and reproduction in any medium, provided the original work is properly cited.

Check for updates

OPEN ACCESS

thrombosis, cerebral venous thrombosis, carotid or vertebral dissections. Single cases of symptomatic cluster headache were described in association with neoplastic conditions like pituitary tumors, meningiomas, glioblastoma, hemangiopericytoma, nasopharynx carcinoma, angiomyolipoma, epidermoid tumor, inflammatory myofibroblastic tumor and lipoma. Nonneoplastic conditions which may cause cluster-like headache include arachnoid cysts, sinusitis, aspergilloma, granolomatous pituitary involvement, orbital pseudotumor, cervical spinal epidural abscess, multiple sclerosis lesions, foreign bodies in the maxillary sinus, cervical syringomyelia and Arnold-Chiari malformation and sarcoidosis. In the last 5 years other possible causes of symptomatic cluster headache were described, including acute rhinosinusitis [4], Sphenoid Ridge Meningioma [5] and middle meningeal artery dural arteriovenous fistulas [6]. Cases associated with rhinosinusitis showed laboratory and radiological aspects of acute inflammation of nasal structures and were always resolved with the efficacious treatment of the inflammatory condition of nasal and para-nasal structures.

\section{Case report}

We describe the case of an 82 years old woman complaining, since one year, of daily left periorbital headache associated with ipsilateral tearing, red eye, rhinorrhea, lasting about one hour. 
Brain magnetic resonance imaging (MRI) showed normal pressure hydrocephalus. The eritrocyte sedimentation rate (ESR) was high (100) without any possible evident reason. No other alteration of laboratory tests was found. She had not a history of cigarette smoke nor familiarity for cluster headache. While she was hospitalized, during a headache bout, it was observed that oxygen inhalation was able to solve in few minutes the attack. Since then, each attack was efficaciously treated with oxygen.

No arterial or venous intracranial alteration was found at angioMRI. Steroid treatment reduced ESR with a reduction of headache attacks of about a half. As previously reported, the MRI scan showed a clear aspect of normal pressure hydrocephalus that was in line with a series of other symptoms that the patient complained of since about one year, including gait disturbances, a mild urinary incontinence and an inconsistent reduction of cognitive performances even if without interference with her daily life activities and selfcare.

When a lumbar puncture was performed and 30 milliliters of cerebrospinal fluid were removed, gait and incontinence improvedand, in parallel, headache disappeared. She was asymptomatic for three months. Three months after discharge, gait and urinary disturbances appeared again, and in parallel headache increased notwithstanding with steroid therapy prosecution, although at low dose. A second lumbar puncture was performed with the same CSF removal and the same positive effect was observed on both headache and NPH syndrome even if steroid dosage was unchanged. ESR was always over $50 \mathrm{~mm} / \mathrm{h}$ (from 89 to 70 during the first hospitalization and 64 during the second).

\section{Discussion and conclusions}

Notwithstanding the age of onset and the female gender are not typical for a primary form of cluster headache, clinical manifestations, attack duration, oxygen inhalation response and close one-sidedness of the symptoms, always on the same side, converge on a diagnosis of cluster headache, probably of symptomatic type. In fact, the atypical age at onset, the effect of CSF removal in preventing headache bouts, and the vascular and hydrocephalic imaging of the brain put in doubt the hypothesis of a primary form, also in line with the point $\mathrm{E}$ of ICHD III diagnostic criteria for cluster headache (Not better accounted for by another ICHD-3 diagnosis).

The diagnosis of NPH was clear: MRI scan of the brain with the typical features, a CSF opening pressures of $220 \mathrm{~mm} \mathrm{H2O}$ (in both lumbar puncture) and a significant improvement of gait and speed at the tap test. For the possible diagnosis of Giant Cell Arteritis, American College of Rheumatology classification criteria were used. The presence of 3 or more out of the five criteria yields a diagnostic sensitivity of $93.5 \%$ and specificity of $91.2 \%$. The five diagnostic elements considered are: 1 . Age 50 years or older; 2 . New-onset localized headache or localized head pain; 3. Temporal artery tenderness to palpation or decreased pulsation; 4 . ESR of $50 \mathrm{~mm} / \mathrm{h}$ or higher; 5. Positive arterial biopsy results (vasculitis characterized by mononuclear infiltration or granulomatous inflammation, usually with multinucleated giant cells). In our patient three out of the five points were present: she was 82 years old; the temporal artery of the painful side was pulseless and ESR was higher than $50 \mathrm{~mm} / \mathrm{h}$. actually, the onset of the headache was not recent, but sub-acute forms are giant cell arteritis are not uncommon [7].

In our knowledge, nor normal pressure hydrocephalus neither arteritis are known causes of symptomatic cluster headache.

A majority of the cases of described symptomatic cluster headaches of the scientific literature had a non-typical presentation in term of atypical symptomatology and abnormal examination (including Horner's syndrome). A significant proportion of symptomatic cluster headache cases were secondary to diseases of the pituitary gland or pituitary region [8] or they were associated with arterial dissection [3], or nasal/paranasal structures [4]. This observation induces to hypothesize that symptoms are generated by the activation/ irritation of neural structures of these intracranial areas and structures.

A possible explanation of the relationship between a normal pressure hydrocephalus and the presence of a symptomatic cluster-like headache may be the effect of cerebral hypertension on the pineal region, but the strictly sided effect seems not to be explainable with this hypothesis. The co-presence of a possible giant cells arteritis (headache of new onset in a geriatric patient with elevated inflammatory markers) may be the key of interpretation of this specific sided effect: the contemporary presence of a sub-acute vascular inflammation of intracranial vessels, associated with an alteration of the pineal region (frequently linked with symptomatic cluster headache) may induce a peculiar kind of headache, atypical for an old woman. Also, the necessity of both steroid therapy and reduction of intracranial pressure with lumbar puncture to prevent recurrence of headache attacks seems to converge on the necessity of both conditions to induce the clinical picture.

In conclusion, this case reinforces the idea that when we observe a typical headache in an atypical patient, it seems to be mandatory a detailed exclusion of all possible causes that have to be removed or treated to obtain the resolution of the problem and to reduce the possibility to treat the symptom and not the cause. Additionally, the efficacious effect of oxygen inhalation on cluster headache attack that frequently is considered as hallmark of primary form, as seen may be present also in secondary cases and has not to be excessively taken in account during the differential diagnosis between 
primary and secondary forms. About this last concept, it is curious to note that the effect ofoxygen on cluster headache was discovered in 1952 by Bayard Taylor Horton (18951980), thesame scientist that givesthe name to the giant cell arteritis of the temporal artery. The mechanismof oxygen efficacy in clusterheadache is not very clear, but some evidence found a certainrelationship to the trigeminovascularsystem and neuroinflammation, two relevant elements also ingiant cell arteritis [9].

\section{References}

1. Manzoni GC, Camarda C, Genovese A, Quintana S, Rausa F, et al Cluster headache in relation to different age groups. Neurol Sci. 2019; 40: 9-13.

PubMed: https://www.ncbi.nlm.nih.gov/pubmed/30796624

2. Rozen TD, Fishman RS. Cluster Headache in the United States of America: Demographics, Clinical Characteristics, Triggers, Suicidality, and Personal Burden. Headache. 2012; 52: 99-113.

PubMed: https://www.ncbi.nlm.nih.gov/pubmed/22077141

3. Edvardsson B. Symptomatic cluster headache: A review of 63 cases. SpringerPlus. 2014; 3: 64.

PubMed: https://www.ncbi.nlm.nih.gov/pubmed/24570848
4. Balgetir $\mathrm{F}, \mathrm{Avcl} \mathrm{D}$, Gönen $\mathrm{M}$, Taşcl I. Acute Rhinosinusitis as an Infrequent Cause of Symptomatic Cluster Headache: Report of Seven Cases. J Oral Facial Pain Headache. 2019; 33: 408-412.

PubMed: https://www.ncbi.nlm.nih.gov/pubmed/31247057

5. Kou L, Huang J, Xu Y, Han C, Ma K, et al. Cluster-like headache secondary to anamnesis of sphenoid ridge meningioma: A case report and literature review. Front Neurol. 2019; 10: 23.

PubMed: https://www.ncbi.nlm.nih.gov/pubmed/30740086

6. Chang YH, Luo CB, Wang SJ, Chen SP. Cluster headache and middle meningeal artery dural arteriovenous fistulas: A case report. Cephalalgia. 2018; 38: 1792-1796.

PubMed: https://www.ncbi.nlm.nih.gov/pubmed/29199428

7. Tsetsou S, Michel P, Ribi C, Hirt L, Kawasaki A, et al. Giant cell arteritis: Guidelines of the University Hospital of Lausanne. Rev Med Suisse. 2015; 11: 411-417.

PubMed: https://www.ncbi.nlm.nih.gov/pubmed/25895220

8. De Pue A, Lutin B, Paemeleire K. Chronic cluster headache and the pituitary gland. J Headache Pain. 2016; 17: 23.

PubMed: https://www.ncbi.nlm.nih.gov/pubmed/26969187

9. Guo XN, Lu JJ, Ni JQ, Lu HF, Zhao HR, et al. The role of oxygen in cluster headache. Med Gas Res. 2019; 9: 229-231.

PubMed: https://www.ncbi.nlm.nih.gov/pubmed/31898608 